\title{
MiR-I44 suppresses cell proliferation, migration, and invasion in hepatocellular carcinoma by targeting SMAD4
}

This article was published in the following Dove Press journal:

OncoTargets and Therapy

29 July 2016

Number of times this article has been viewed

Min Yu*

Ye Lin*

Yu Zhou

Haosheng Jin

Baohua Hou

Zhongshi Wu

Zhide Li

Zhixiang Jian

Jian Sun

Department of General Surgery, Guangdong General Hospital, Guangdong Academy of Medical Sciences, Guangzhou, Guangdong, People's Republic of China

*These authors contributed equally to this work

Correspondence: Jian Sun Department of General Surgery, Guangdong General Hospital, Guangdong Academy of Medical Sciences, Guangzhou, Guangdong 510080 , People's Republic of China Email sunyat1877@।63.com
Background/aim: Increasing evidence show microRNAs (miRNAs) are engaged in hepatocellular carcinoma (HCC). The aim of this study was to investigate the role of miR-144 in HCC, as well as to identify its underlying mechanism.

Methods: The expression levels of miR-144 were assessed in multiple HCC cell lines, as well as in liver tissues from patients with HCC. We further examined the effects of miR-144 on HCC. The molecular target of miR-144 was identified using a computer algorithm and confirmed experimentally.

Results: We found that the levels of miR-144 were frequently downregulated in human HCC tissues and cell lines, and overexpression of miR-144 dramatically inhibited HCC metastasis, invasion, cell cycle, epithelial-mesenchymal transition, and chemoresistance. We further verified the SMAD4 as a novel and direct target of miR-144 in HCCs.

Conclusion: Taken together, overexpression of miR-144 or downregulation of SMAD4 may prove beneficial as therapeutic strategies for HCC treatment.

Keywords: microRNA, liver cancer, therapeautic target

\section{Introduction}

Hepatocellular carcinoma (HCC) is one of the most prevalent malignant diseases and the third leading cause of cancer-related deaths. One-half of the new HCC cases and HCC deaths worldwide were estimated to occur in the People's Republic of China. ${ }^{1}$ Currently, surgical resection, liver transplantation, and radiofrequency ablation are the effective approaches for HCC treatment. The recurrence rate of HCC within 2 years in patients who received surgery exceeds $50 \%$. Due to the late detection of the tumors and high rate of recurrence and metastasis, the prognosis of HCC is still dismal, and the 5-year survival rate for patients is less than $5 \% .^{2}$ Therefore, further elucidation of the molecular mechanisms underlying HCC invasion and metastasis are important for the development of new therapeutic strategies for diagnosis, treatment, and prognosis of HCC.

MicroRNAs (miRNAs) are a class of small, short, and noncoding RNAs, regulating gene expression by binding to sequences in a $3^{\prime}$-untranslated region ( $3^{\prime}$-UTR) of the target mRNA, resulting in upregulation and downregulation of the targeted gene. ${ }^{3,4}$ In various human cancers, some miRNAs are often upregulated and have an oncogenic function, while most miRNAs are downregulated and may possess a tumorsuppressive activity. Accumulating evidence suggests that the abnormal expression of miRNAs is involved in the invasion and metastasis during the progression of various human cancers. 
Recent evidence indicates that miRNA expression profiling has been characterized in a variety of cancers, including breast cancer, ${ }^{5}$ pancreatic cancer ${ }^{6}$ ovarian cancer, ${ }^{7}$ and HCC. ${ }^{3}$ Previous data showed that certain miRNAs are involved in the proliferation and survival of HCC, including miR-199, miR-7, miR-124, and so on. In this study, we found that the levels of a specific miRNA, miR-144, were frequently downregulated in human HCC tissues and cell lines, and overexpression of miR-144 dramatically inhibited HCC metastasis, invasion, cell cycle, epithelial-mesenchymal transition, and chemoresistance. We further verified the SMAD4 as a novel and direct target of miR-144 in HCCs. In summary, our data demonstrate that SMAD4 expression is inversely correlated to miR-144 levels in HCC tissues and cell lines, and that overexpression of miR-144 in HCC cell lines decreases $S M A D 4$ mRNA and protein levels by directly binding to the 3'-UTR of SMAD4, which subsequently leads to downregulation of SMAD4. Therefore, our data strongly indicate that miR-144 is a tumor suppressor by targeting SMAD4 expression to modulate HCC biological behaviors. Taken together, overexpression of miR-144 or downregulation of SMAD4 may prove beneficial as therapeutic strategies for HCC treatment.

\section{Methods}

\section{Patient selection}

Samples of $100 \mathrm{HCC}$ tissues were obtained from patients who had undergone HCC surgical resection at the Guangdong General Hospital. The study complied with the Declaration of Helsinki and was approved by the Institutional Ethics Committee of Guangdong General Hospital. All patients signed consent forms indicating their willingness to participate, and their understanding of the procedure and general aim of the study. All of the included patients met the following criteria: pathologically and histologically confirmed HCC, no history of any other malignant tumors, and no neoadjuvant therapy prior to the surgery.

\section{Cell culture}

The following human $\mathrm{HCC}$ cell lines were studied: MHCC-97H, SMMC-7221, HepG2, Huh-7, and Hep3B. The normal human liver LO2 cell line was also employed as normal control. All cells were grown in Dulbecco's Modified Eagle's Medium (Thermo Fisher Scientific, Waltham, MA, USA) and supplemented with $10 \%$ fetal bovine serum (HyClone, Logan, UT, USA) and 1\% penicillin/ streptomycin.

\section{Manipulation of miR-I44 expression levels}

The miR-144 mimics and negative control (micrONTM miRNA Mimic Negative Controls) were purchased from Land (Guangzhou, Guangdong, People's Republic of China). The final concentration of transfection is $50 \mathrm{nM}$.

\section{Cell transfections}

Transfection of the miR-144 mimics was performed using Lipofectamine ${ }^{\circledR}$ RNAiMAX (Thermo Fisher Scientific) according to the manufacturer's instructions.

\section{RNA extraction and real-time PCR analysis}

Total RNA was extracted from the cell lines and frozen tissue specimens with TRIzol reagent (Thermo Fisher Scientific), and the concentration of the total RNA was quantitated by measuring the absorbance at $260 \mathrm{~nm}$. Complementary DNA was generated using a miScript Reverse Transcription Kit (Qiagen NV, Venlo, the Netherlands). Primers for miR-144 and the U6 small nuclear RNA (snRNA) (internal control) were purchased from Land. The expression level of miRNA was defined based on the threshold cycle $(\mathrm{Ct})$, and relative expression levels were calculated using the $2^{-\Delta \Delta \mathrm{Ct}}$ method, using the expression level of the U6 snRNA as a reference gene. Each polymerase chain reaction (PCR) was performed in triplicate. The primers for the examined genes are presented in Table 1.

\section{Cell invasion assay}

The invasion assay was performed using a transwell chamber, consisting of $8 \mathrm{~mm}$ membrane filter inserts (Corning Incorporated, Corning, NY, USA) coated with Matrigel (BD Biosciences, San Jose, CA, USA). Briefly, cells were trypsinized and suspended in serum-free medium. Next, $1.5 \times 10^{5}$ cells were added to the upper chamber, and the lower chamber was filled with medium containing $10 \%$ fetal bovine serum. After 36 hours of incubation, cells that had invaded the lower chamber were fixed with $4 \%$ paraformaldehyde, stained with hematoxylin, and counted using a microscope.

Table I Primer for qRT-PCR

\begin{tabular}{ll}
\hline hsa-miR-I44 & F: 5'-ACACTCCAGCTGGGTACAGTATAGATGATGTA \\
U6 & R: 5'-CTCAACTGGTGTCGTGGA \\
& F: 5'-CTCGCTTCGGCAGCACA \\
& R: 5'-AACGCTTCACGAATTTGCGT \\
\hline
\end{tabular}

Abbreviation: qRT-PCR, quantitative reverse transcription polymerase chain reaction. 


\section{Wound-healing assay}

Wound-healing assay was performed using HepG2 and Huh-7 cells. Cells were trypsinized and seeded in equal numbers into six-well tissue culture plates, and allowed to grow until confluent (approximately 24 hours). Following serum starvation for 24 hours, an artificial homogenous wound (scratch) was created onto the cell monolayer with a sterile $100 \mu \mathrm{L}$ tip. After scratching, the cells were washed with serum-free medium, complete media was added, and microscopic images (20× magnification) of the cells were collected at 0,12 , and 24 hours.

\section{Luciferase reporter assay}

Luciferase reporter assay was performed according to the manufacturer's instructions. Briefly, cells $\left(3.5 \times 10^{4}\right)$ were seeded in triplicate in 24-well plates overnight. Next, $100 \mathrm{ng}$ of pGL3-SMAD4-3'-UTR (wild type/mutant) or controlluciferase plasmid plus $1 \mathrm{ng}$ of pRL-TK renilla plasmid (\#E2810; Promega, Madison, WI, USA) were transfected into the cells using Lipofectamine ${ }^{\circledR} 2000$ (Thermo Fisher Scientific). Three independent experiments were performed and the data are presented as the mean \pm standard deviation (SD).

\section{Statistical analysis}

A Student's $t$-test was used to evaluate the statistical significance of the difference between two groups of data. $P$-value of less than 0.05 was considered to be statistically significant. All analyses in the present study were performed by SPSS 13.0 (SPSS Inc., Chicago, IL, USA) statistical software package.

\section{Results}

\section{The expression of miR-I44 is frequently downregulated in HCC cell lines and tissues}

To determine whether miR-144 is correlated with the progression of HCC, the expression level of miR-144 was detected in HCC cell lines, tissues, and matched with adjacent nontumor liver tissues obtained from 100 patients by quantitative reverse transcription polymerase chain reaction (qRT-PCR). The results showed that the expression of miR-144 was dramatically decreased in various HCC cell lines, including MHCC-97H, SMMC-7221, HepG2, Huh-7, and Hep3B, compared with the normal hepatic cell line LO2 (Figure 1A). As shown in Figure 1B, the expression of miR-144 was found decreased (negative expression and low expression of miR-144) in $85.0 \%(85 / 100)$ of $\mathrm{HCC}$ tissues compared with matched adjacent nontumor liver tissues, with an average of 5.20-fold reduction in expression (median $=0.73$ vs $1.52 ; P<0.01$ ). No statistically significant relationships were found between miR-144 expression and any of the clinicopathological parameters except for recurrence $(P=0.0041)$ (Table 2$)$. Moreover, the expression of miR-144 was significantly higher in the HCC samples at early stages (TNM I and II) compared to that of the HCC samples at advanced tumor stages (TNM III) (Figure 1C).

\section{Effect of miR-I44 on the proliferation of HCC cells}

To determine whether miR-144 can inhibit the proliferation of HCC cells, miR-144 mimics was transfected into HepG2 and MHCC-97H cells. EdU analysis of S-phase cells showed that EdU-positive cells decreased significantly after treatment with miR-144 mimics compared with mimic mock control in both MHCC-97H $(45.68 \%$ vs $27.53 \%, P<0.001)$ and HepG2 (49.67\% vs $23.46 \%, P<0.001)$ (Figure $2 \mathrm{~A}$ and $\mathrm{B}$ ). MTT assays indicated that both cells with miR-144 mimics proliferated at a slower rate than did control cells, and statistical analysis showed a significant difference after culture for 4 days (Figure 2C and D). The cell cycle of these cells was detected by flow cytometry. The results showed that $32.70 \%$ of MHCC-97H and $32.74 \%$ of HepG 2 cells were in S-phase, while $22.96 \%$ of MHCC-97H and $23.15 \%$ of HepG2 cells were in S-phase after treatment with miR-144 mimics $(P<0.05$, Figure $2 \mathrm{E}-\mathrm{G})$. Therefore, the results indicated that miR-144 can suppress the cell cycle progression and inhibit the proliferation of HCC cells.

\section{Effect of miR-I 44 on migration and invasion of HCC}

To further investigate the biological significance of miR144 in HCC, we detected the effect of miR-144 on migration and invasion of HCC cells. Wound-healing assay showed that the mobility of HepG2 and MHCC-97H cells evidently decelerated in rate in within 48 hours compared with controls (wound closure of $24.71 \%$ vs $100.00 \%$ in MHCC-97H, $P<0.001 ; 24.01 \%$ vs $100.00 \%$ in HepG2 at 48 hours, $P<0.001$ ) (Figure $3 \mathrm{~A}$ and B). Transwell with Matrigel showed that treatment with miR-144 led to a significant decrease in invasive potential of MHCC-97H (6.31-fold reduction, $P<0.001)$ and HepG2 (5.14-fold reduction, $P<0.001$ ) (Figure $3 \mathrm{C}$ and $\mathrm{D}$ ). Taken together, the expression of miR-144 suppresses the migration and invasion of HCC. 
A

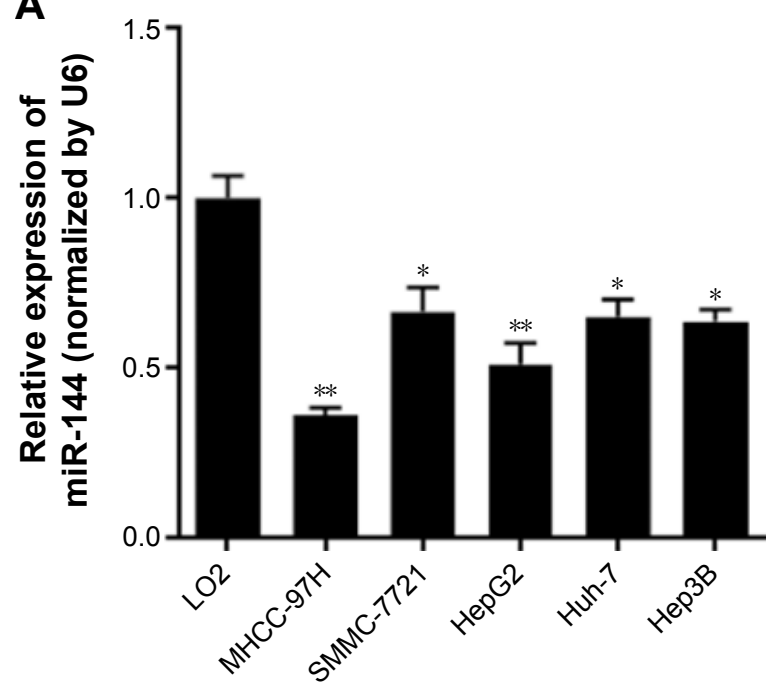

B

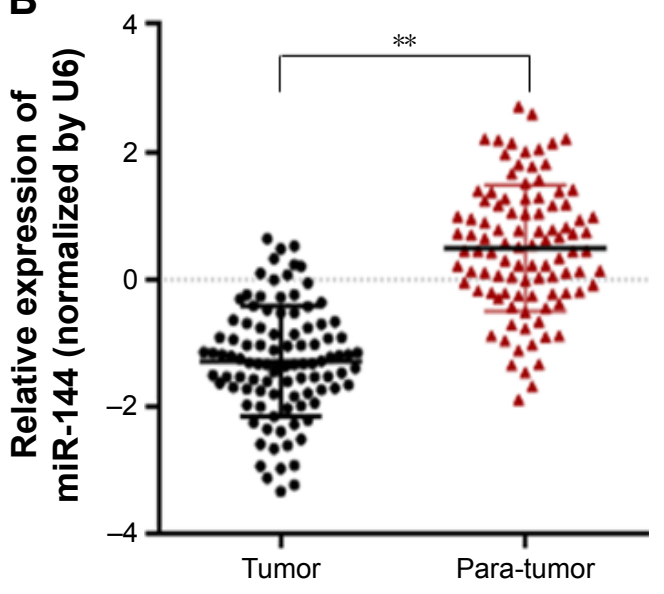

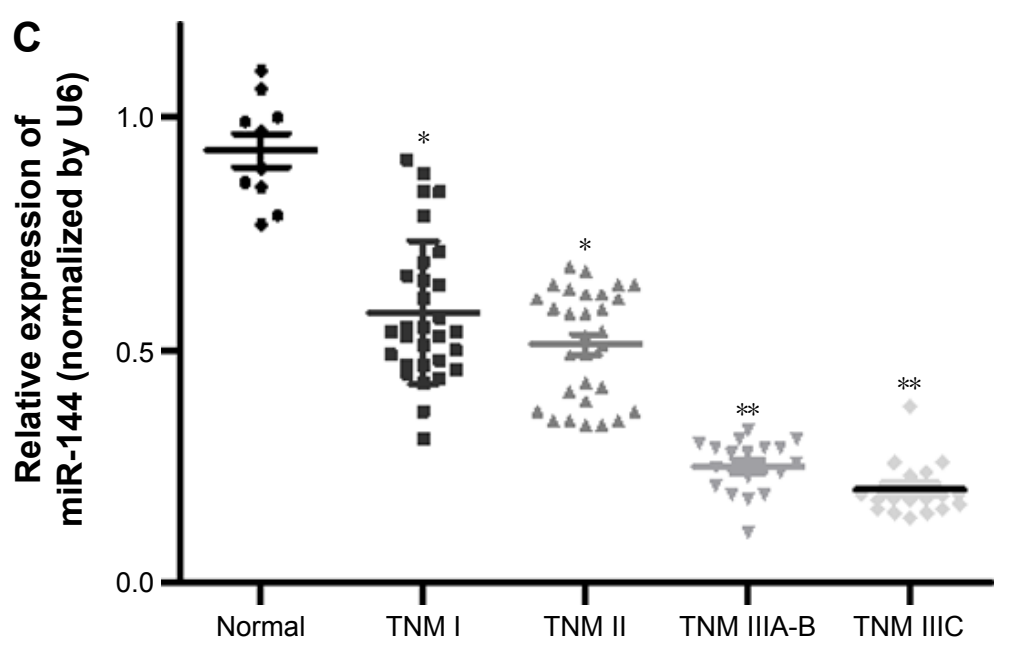

Figure I Downregulation of miR-I44 in HCC tissues and cell lines detected by qRT-PCR.

Notes: (A) The relative miR-I44 expression in HCC cell lines was much lower than the normal liver cell line LO2. The relative expression of miR-I44 was normalized to the endogenous control U6 snRNA. Each sample was analyzed in triplicate. ${ }^{*} P<0.05$, $* * P<0.0$ I, compared with normal liver cell line. (B) The expression level of miR-I44 was decreased in $100 \mathrm{HCC}$ tissues than their matched adjacent nontumor liver tissues. Each sample was analyzed in triplicate and normalized to the U6 snRNA. Data represent mean $\pm \mathrm{SD}$. $* * P<0.0$ l, compared with normal tissues. (C) The relative expression level of miR-26b was examined in normal human liver tissue (Normal), and HCC tissues at different WHO classifications. $* P<0.005 ; * * P<0.01$.

Abbreviations: HCC, hepatocellular carcinoma; GAPDH, glyceraldehyde 3-phosphate dehydrogenase; qRT-PCR, quantitative reverse transcription polymerase chain reaction; snRNA, small nuclear RNA; SD, standard deviation; WHO, World Health Organization.

\section{Effect of miR-I44 on chemoresistance and apoptosis of HCC}

As shown in Figure 4A, 5-fluorouracil (5-FU) inhibited cell proliferation of both cell lines in a dose-dependent manner. The half maximal inhibitory concentration $\left(\mathrm{IC}_{50}\right)$ values of 5-FU were $88.56 \pm 43.29$ and $10.19 \pm 3.31 \mathrm{mg} / \mathrm{L}$ after transfection by miR-144 in MHCC-97H $(P<0.001)$, whereas $\mathrm{IC}_{50}$ values were $10.66 \pm 2.19$ and $1.92 \pm 0.48 \mathrm{mg} / \mathrm{L}$ after transfection in HepG2 $(P<0.001)$ (Figure 4A and B). We also detected the apoptotic population of both cell lines transfection by miR-144 by flow cytometry. The results showed that $1 \mathrm{mg} / \mathrm{L} 5$-FU-induced apoptotic populations of MHCC-97H and HepG2 were $56.37 \%$ and $60.15 \%$, respectively. After transfection by miR-144, the apoptotic population reduced to $8.48 \pm 0.47(P<0.001)$ and $13.46 \pm 0.52(P<0.001)$ (Figure 4C and D). Our results generally showed that miR-144 can enhance the chemosensitivity in HCC cell lines.

\section{Function of miR-I44 in HCC cells partially attributed to targeting SMAD4}

To determine the underlying mechanism by which miR-144 regulates progression and chemoresistance of $\mathrm{HCC}$, we integrated bioinformatics algorithms, including miRanda, PicTar, and TargetScan, to predict the potential direct target of 
Table 2 Relationship between miR- 444 expression and clinicopathological features of HCC patients

\begin{tabular}{|c|c|c|c|c|}
\hline \multirow[t]{2}{*}{ Clinicopathological parameters } & \multirow[t]{2}{*}{ Number of cases (\%) } & \multicolumn{3}{|c|}{ Expression of miR-I44 } \\
\hline & & High (15/100) & Low $(85 / 100)$ & $P$-value \\
\hline Sex & & & & 1.0000 \\
\hline Male & 48 & 7 & 41 & \\
\hline Female & 52 & 8 & 44 & \\
\hline Age (years) & & & & 0.2591 \\
\hline$\leq 60$ & 56 & 6 & 50 & \\
\hline$>60$ & 44 & 9 & 35 & \\
\hline Diameter $(\mathrm{cm})$ & & & & 1.0000 \\
\hline$\leq 5.0$ & 60 & 9 & 51 & \\
\hline$>5.0$ & 40 & 6 & 34 & \\
\hline Histological differentiation & & & & 0.1315 \\
\hline Well & 35 & 2 & 33 & \\
\hline Moderate & 49 & 9 & 40 & \\
\hline Poor & 16 & 4 & 12 & \\
\hline Liver cirrhosis & & & & 0.8191 \\
\hline Yes & 35 & 6 & 29 & \\
\hline No & 65 & 10 & 55 & \\
\hline Recurrence & & & & $0.004 I^{*}$ \\
\hline Yes & 29 & 9 & 20 & \\
\hline No & 71 & 6 & 65 & \\
\hline HBsAg status & & & & 0.6360 \\
\hline Positive & 78 & II & 67 & \\
\hline Negative & 22 & 4 & 18 & \\
\hline Serum AFP (ng/mL) & & & & 0.9147 \\
\hline$<25$ & 19 & 3 & 16 & \\
\hline$\geq 25$ & 81 & 12 & 69 & \\
\hline
\end{tabular}

Note: *Statistically significant $(P<0.05)$.

Abbreviations: AFP, $\alpha$-fetoprotein; HBsAg, hepatitis B surface antigen; HCC, hepatocellular carcinoma.

A MHCC-97H
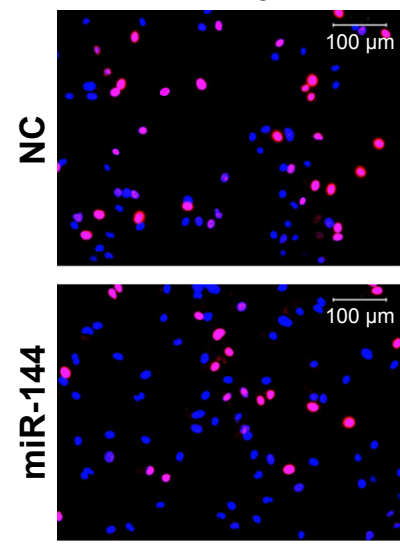

C

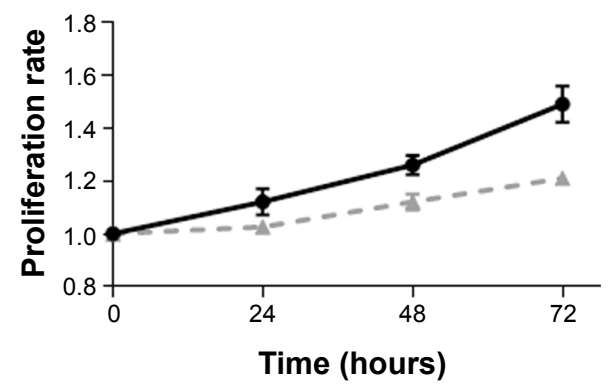

HepG2
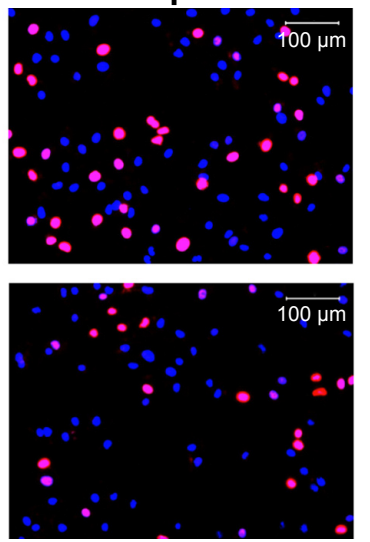

MHCC-97H

$\leadsto \mathrm{NC} \quad-\mathrm{A}=\mathrm{miR}-144$
B

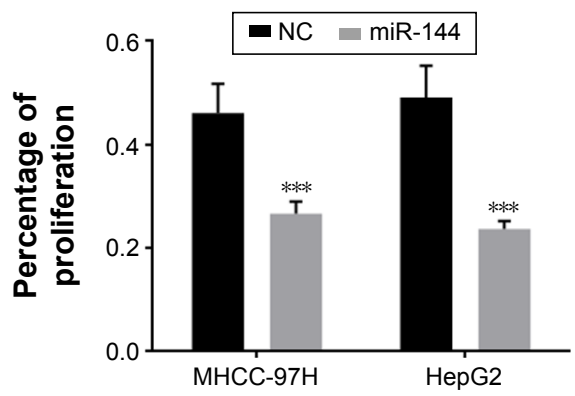

HepG2

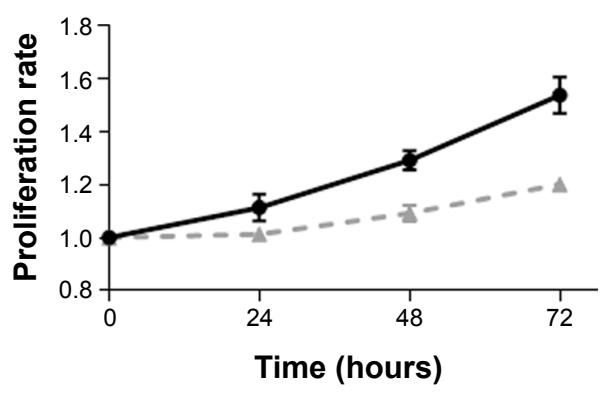

Figure 2 (Continued) 

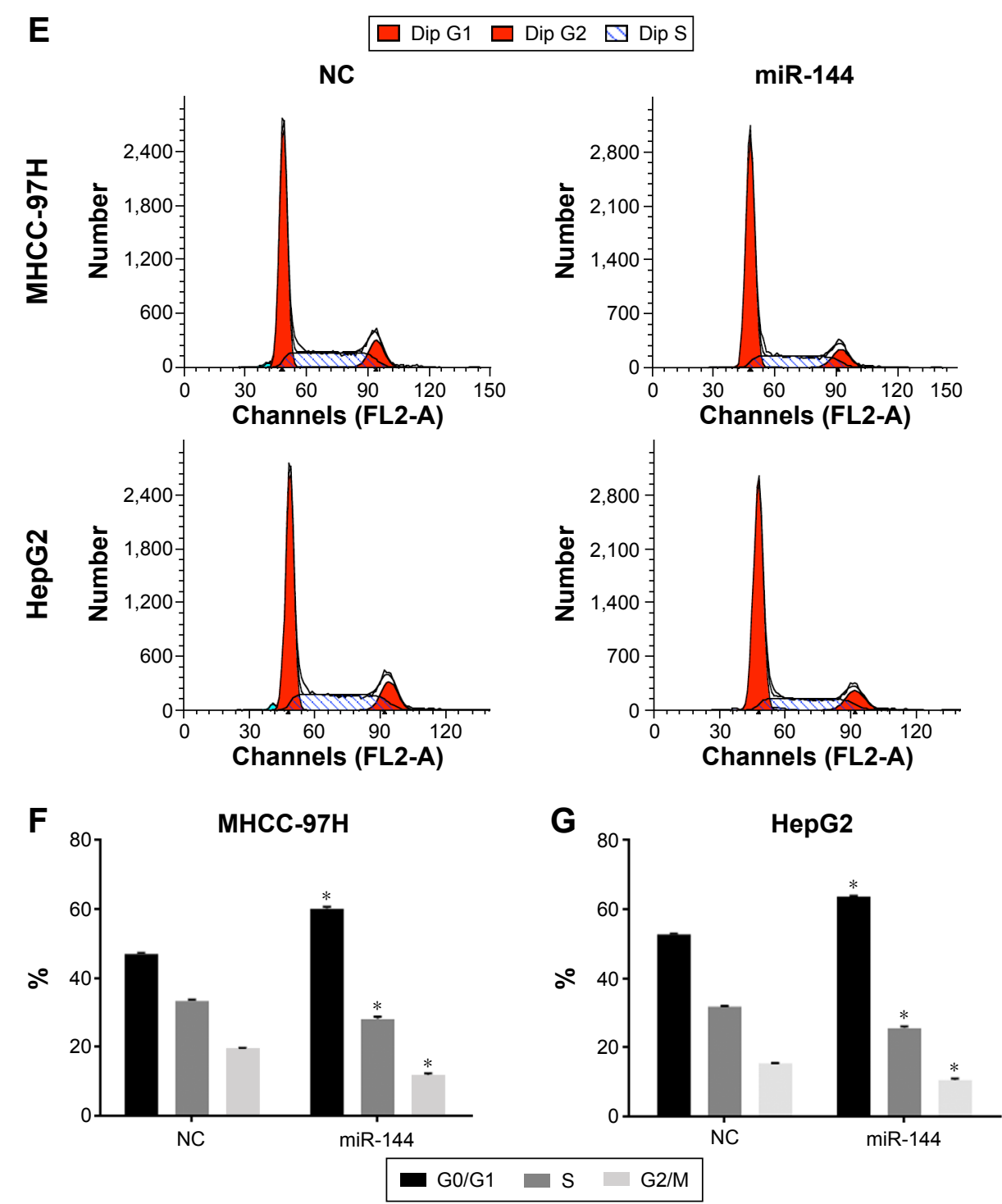

Figure 2 Effect of miR-I44 on the proliferation of HCC cells.

Notes: (A) miR-144 decreased HCC cell line MHCC-97H and HepG2 in S-phase. Blue color represents the nucleus and red color indicates S-phase cells (EdU-positive). (B) Histological analysis of the percent of EdU-positive cells in mimic mock control and miR-144 mimics in HCC cell lines. Data are presented as mean $\pm \mathrm{SD}$ ( $\mathrm{n}=3$ ). $* P<0.05$, $* * * P<0.00$ I between control cells and cells with miR-144 mimics. (C, D) Proliferation rates were determined by MTT assays after transfection of the miR-I44 mimics and mimic mock control in MHCC-97H (C) and HepG2 (D) cell lines. miR-I44 inhibited cell proliferation in both cell lines compared with the controls. (E-G) Representative images of the cell cycle distribution of MHCC-97H and HepG2 by flow cytometry (E). Cycle phase distributions (percentage) were determined by FACS analysis and plotted. Both cells were treated with miR-I44 mimics and mimic mock as vehicle control for 24 hours. Data are presented as mean $\pm S D(n=3)$. $* P<0.05$.

Abbreviations: HCC, hepatocellular carcinoma; SD, standard deviation; FACS, fluorescence-activated cell sorting; NC, negative control.

miR-144. According to the prediction, SMAD4 has the putative miR-144-binding site that maps to the $3^{\prime}$-UTR. To further validate the prediction results, we constructed the luciferase reporters carrying the wild type and mutant type of SMAD4 3'-UTR (Figure 5A). As shown in Figure 5B, luciferase assays indicated that the wild type of 3 '-UTR caused a significant reduction in luciferase activity, whereas mutation of the key seed region in the 3 '-UTR of SMAD4 showed no variations in the luciferase activity compared with the control (Figure 5B). The qRT-PCR analysis suggested that treatment by miR-144 mimics significantly repressed the expression of SMAD4
mRNA (Figure 5C). These findings were further verified by Western blot analysis, which indicated that treatment by miR-144 mimics markedly inhibited SMAD4 protein level (Figure 5D). Taken together, these results strongly suggested that miR-144 could significantly suppress the expression of SMAD4 through targeting the 3'-UTR.

To determine the correlation of miR-144 and SMAD4 expression in clinical HCC tissues, qRT-PCR was employed to assess the expression of SMAD4 in 100 HCC tissues. As indicated in Figure 5E, results of Spearman's rank test showed a significantly negative correlation between miR-144 
A

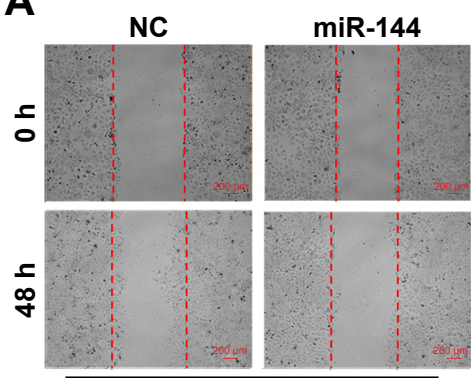

MHCC-97H
NC

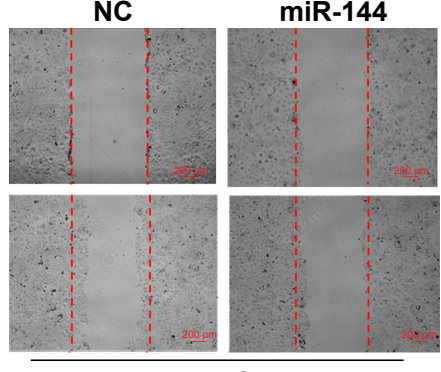

B

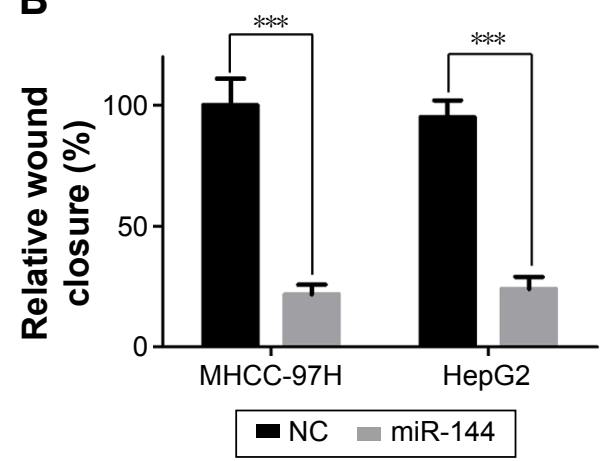

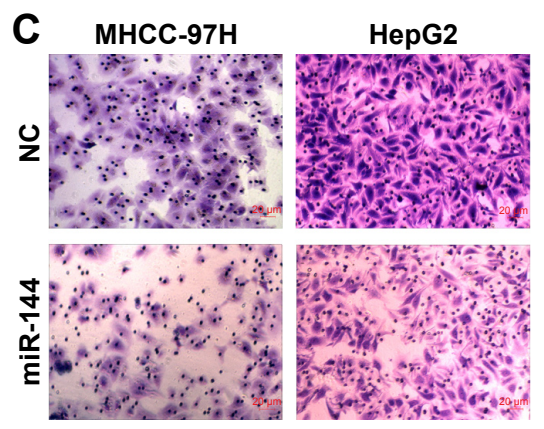

D

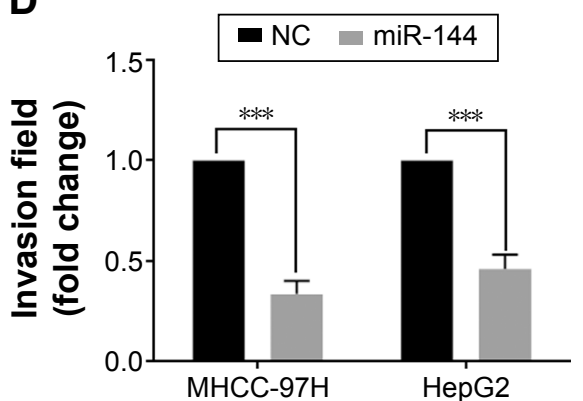

Figure 3 Effect of miR-144 on growth and invasion of $\mathrm{HCC}$ cell in vitro.

Notes: (A, B) Representative images of wound-healing assay (A). The wound healing rate (B) in MHCC-97H and HepG2 cell lines transfected with miR-144 mimics was significantly decreased compared with the mimic mock control. (C, D) Representative images (C) and quantification (D) of the transwell invasion assay. The number of invaded cells in the MHCC-97H and HepG2 transfected with miR-I 44 was significantly decreased compared with the mimic mock control. Data are presented as mean \pm SD; $P$-values were calculated using the Student's $t$-test. $* * * P<0.001$.

Abbreviations: HCC, hepatocellular carcinoma; SD, standard deviation; NC, negative control; h, hours.

and SMAD4 expression ( $r=-0.768, P<0.001)$ (Figure 5E). Therefore, our results suggested that miR-144 represses the HCC development of HCC partly through inhibiting the expression of SMAD4.

\section{Discussion}

More and more evidence indicate that miRNAs are important regulators in various cellular processes and are recently extensively investigated relating to cancer initiation, progression, diagnosis, and treatment. ${ }^{8}$ The involvement of miRNAs in cancer pathogenesis is well established, as they behave as oncogenic or tumor-suppressive role depending on their functional targets. ${ }^{9}$ Dysregulation of miRNAs is often found in $\mathrm{HCC}$, and some of them have an important role in the progression and development of $\mathrm{HCC}^{3}$ However, the role of miRNAs in the pathogenesis of HCC is still largely unclear as a single miRNA may regulate multiple target genes and a single mRNA may be regulated by various miRNAs. ${ }^{10}$ Given the complexity of the network between mRNAs and miRNAs, further studies are needed to determine the importance of miRNAs because of the potential in cancer diagnostic and prognostic value. Further understanding of the functional role of miRNAs in cancer helps to better reveal the underlying mechanism of HCC pathogenesis and progression.

Previous evidence demonstrated that miR-144 expression was deceased in various cancers, including HCC, cholangiocarcinoma, ${ }^{11}$ colorectal cancer, ${ }^{12}$ bladder cancer, ${ }^{13}$ and thyroid cancer, ${ }^{14}$ and inversely related with cancer proliferation and metastasis. Although a previous study demonstrated that miR-144 might suppress the growth and motility of HCC cells partially by targeting E2F3, the knowledge about the role of miR-144 in HCC is still limited. ${ }^{15}$ Therefore, the present study was carried out to further investigate the functional role of miR-144 in HCC. The findings of the present study are in line with those of previous evidence, which showed the reduced expression of miR-144 in cancer cell lines and human tissues. The present study showed that decreased expression was found in HCC cell lines and human tissues, and negatively correlated with the severity and progression of $\mathrm{HCC}$, suggesting that decreased expression of miR-144 correlated with the malignant potential of HCC. Therefore, we assessed the effect of miR-144 on proliferation, migration, invasion, chemoresistance, and apoptosis. Consistent with prior studies, ${ }^{15}$ we showed that upregulation 


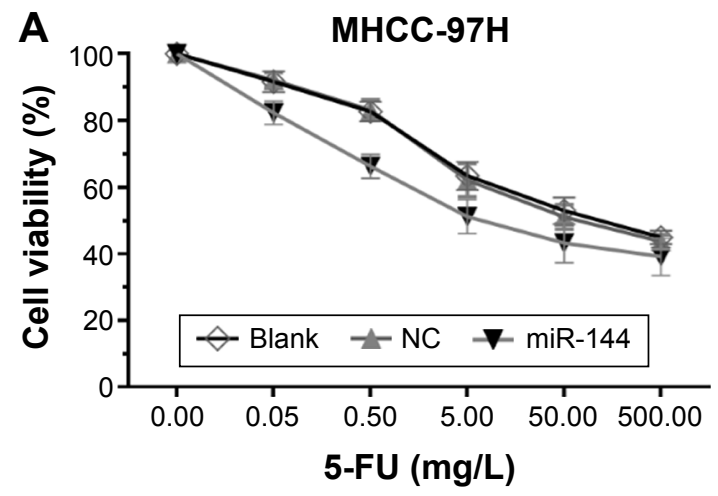

C

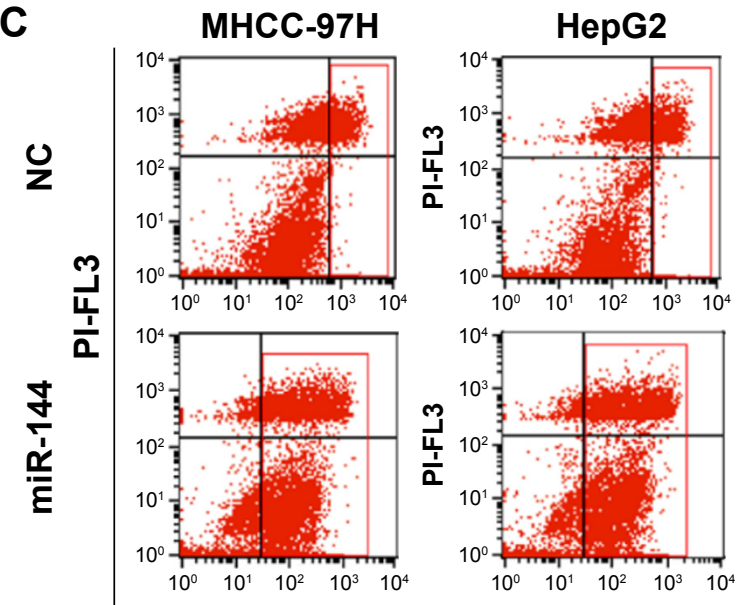

Annexin V FITC

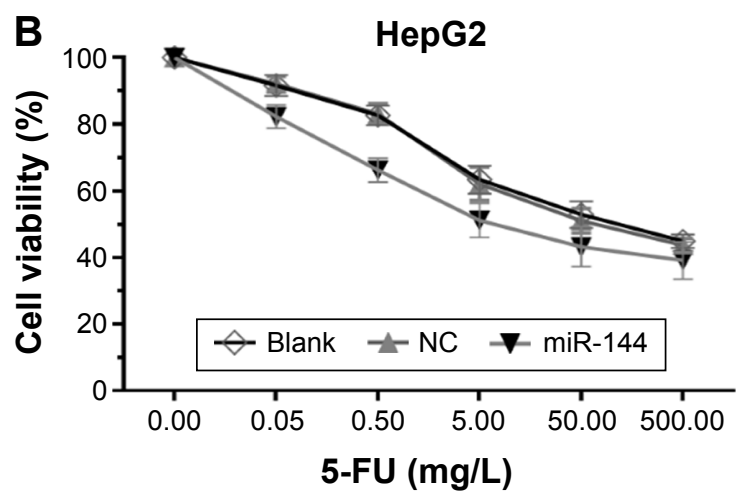

D

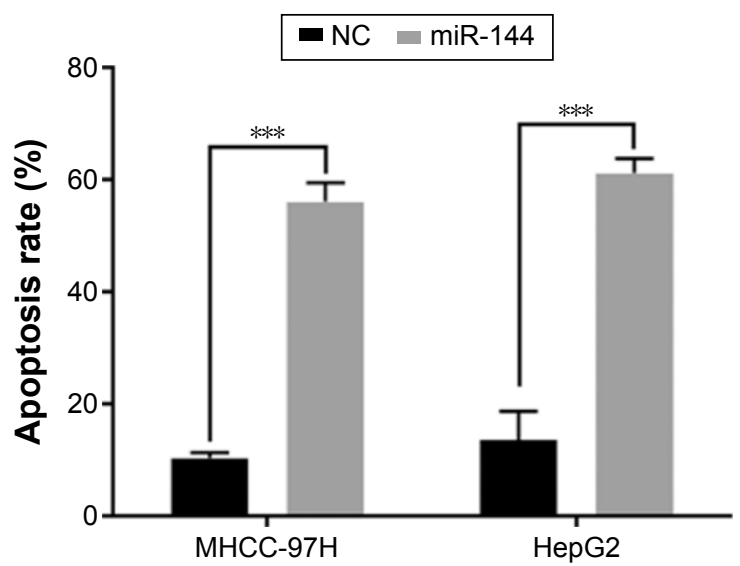

Figure 4 Effect of miR-I44 on chemoresistance and apoptosis of HCC.

Notes: (A, B) Cell viability were determined by MTT assays at 48 hours after transfection of the miR-144 mimics and mimic mock control in MHCC-97H and HepG2 cell lines. (C) Apoptotic population of MHCC-97H and HepG2 cell lines. Flow cytometric analysis of I mg/L 5-FU-induced apoptosis in MHCC-97H and HepG2 cell lines using Annexin V FITC/PI. Quadrants: QI: normal cells; Q2: necrotic cells; Q3: live cells; Q4: apoptotic cells. (D) Columns, mean of three experiments. Data are presented as mean $\pm S D$. $* * * P<0.001, P$-value compared with the control group.

Abbreviations: HCC, hepatocellular carcinoma; SD, standard deviation; FITC, fluorescein isothiocyanate; PI, propidium iodide; NC, negative control; 5-FU, 5-fluorouracil.

A

SMAD4 3'-UTR 5' ...UAAAUUCUAUGUUAAAUACUGUG...

|| ||||

has-miR-144-3p 3' UCAUGUAGUAGAUAUGACAU

SMAD4 3'-UTR mutant ...UAAAUUCUAUGUUAAUAUGUGUG...

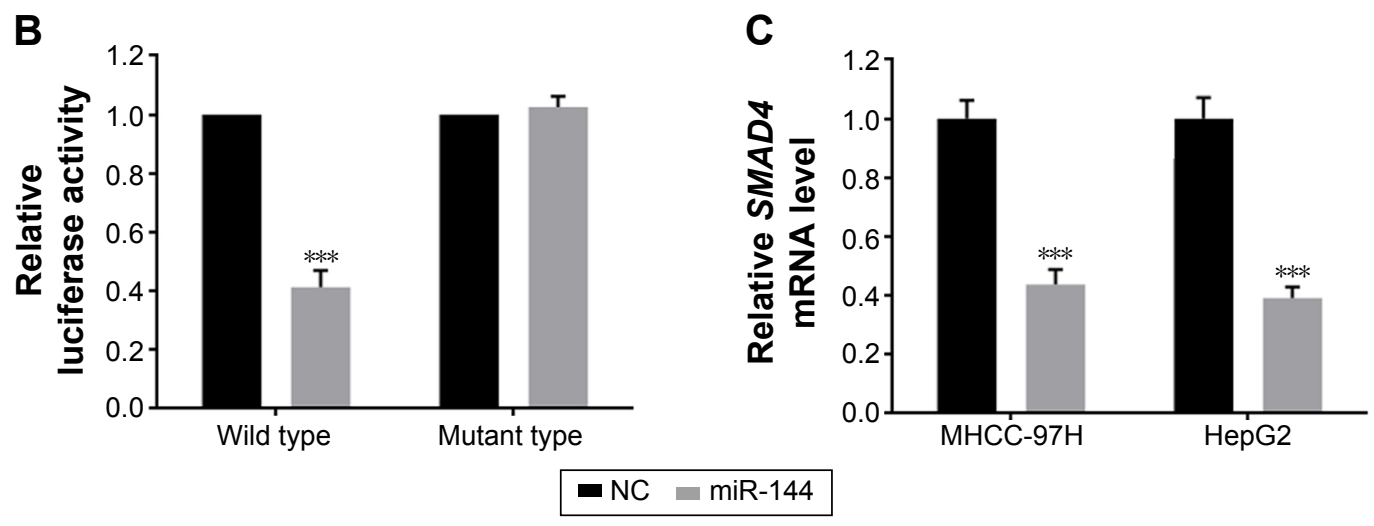

Figure 5 (Continued) 

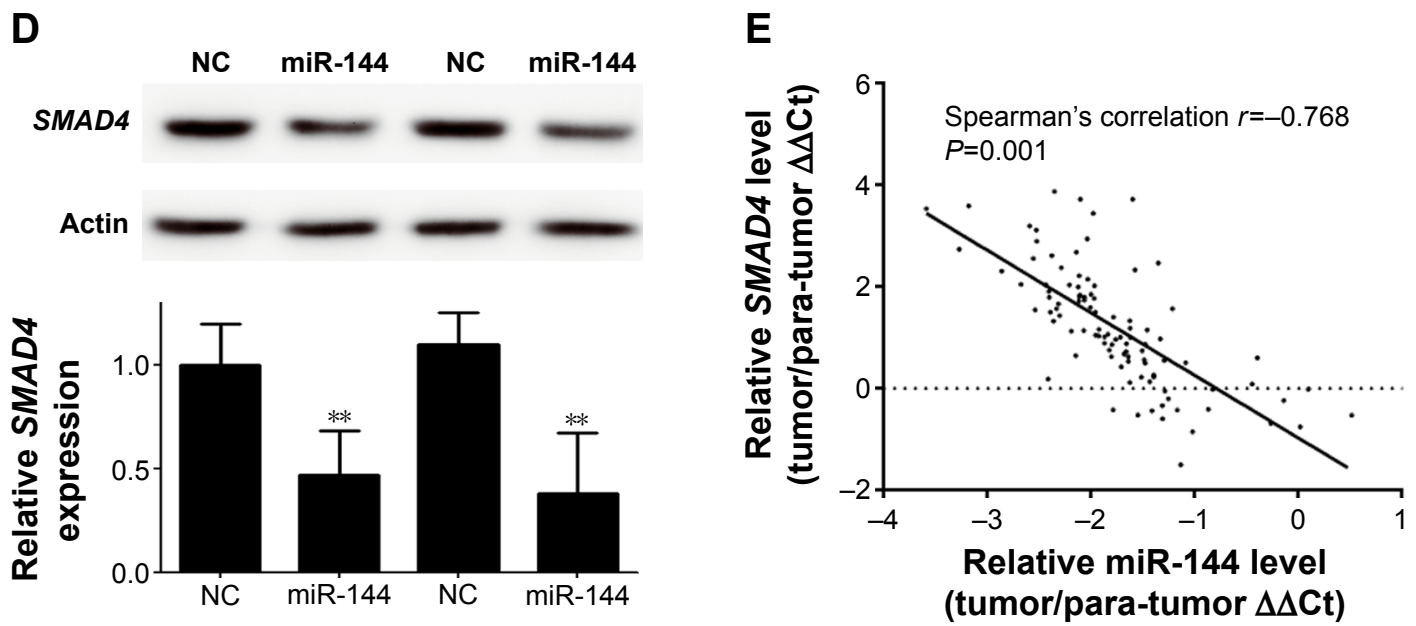

Figure 5 Function of miR-I44 in HCC cells partially attributed to targeting SMAD4.

Notes: (A) miR-144 and its putative binding sequence in the 3'-UTR of SMAD4. The mutant SMAD4 binding site was generated in the complementary site for the seed region of miR-144. (B) miR-I44 significantly suppressed the luciferase activity that carried wild-type SMAD4 but not the mutant SMAD4. (C) Overexpression of miR-I44 significantly decreased the mRNA levels of SMAD4 in MHCC-97H and HepG2 cell lines compared with control. (D) Overexpression of miR-144 significantly decreased the protein levels of SMAD4 in MHCC-97H and HepG2 cell lines compared with control. (E) Analysis of correlation of miR-I44 and SMAD4 expression in HCC tissues (two-tailed Spearman's correlation analysis, $r=-0.768 ; P=0.00 \mathrm{I}, \mathrm{n}=100$ ). Data were presented as fold change of $\mathrm{HCC}$ tissues relative to nontumor adjacent tissues. $* * P<0.0 \mathrm{I} ; * * * P<0.00 \mathrm{I}$.

Abbreviations: HCC, hepatocellular carcinoma; UTR, untranslated region; 5-FU, 5-fluorouracil; NC, negative control; Ct, threshold cycle.

of miR-144 can suppress the proliferation, migration, and invasion of HCC cell lines. Further, our results revealed that upregulation of miR-144 could repress cell cycle progression by inducing G0/G1 cell cycle arrest, and also could enhance chemosensitivity and induced cancer cell apoptosis. Also, we identified SMAD4 as a novel target of miR-144 in HCC cells. SMAD4 proved to be the key mediator of transforming growth factor beta (TGF- $\beta$ ) pathway, ${ }^{16-18}$ which has a central role in the growth of hepatocytes. ${ }^{19}$ Interestingly, $\mathrm{Xu}$ et al reported that miR-144 is a critical regulator of the TGF- $\beta$ signaling cascade and is overexpressed in lungs with bronchiolitis obliterans syndrome, which suggest an important role of miR-144 in regulating TGF- $\beta$ pathway. ${ }^{20}$ According to the previous data, SMAD4 has dual roles of tumor-suppressive and tumor-promoting effects in different cancers. Loss or inactivation of SMAD4 is proved to be inversely related with prognosis pancreatic cancer, ${ }^{21,22}$ colorectal cancer, ${ }^{21}$ cholangiocarcinoma, ${ }^{23}$ and other malignancies. ${ }^{24}$ However, increased expression of SMAD4 was observed in $\mathrm{HCC}^{16,25,26}$ and correlated with poor prognosis. ${ }^{27}$

Recent evidence suggested that SMAD4 processes a highly tumor-promoting function of SMAD4 in HCC and might serve as an ideal therapeutic target. Therefore, SMAD4 inhibition represents a rational and promising new approach for HCC therapy due to its unique and specific role in HCC. To validate the prediction experimentally, luciferase reporter assay was employed and the results confirmed that SMAD4 is a target gene of miR-144. These data were further strengthened by assessment of the protein level of SMAD4 in both
HCC cell lines treated with miR-144 mimics. Moreover, the coexpression of miR-144 and SMAD4 was detected in HCC tissues, and the results showed a significantly negative correlation between them. Taken together, these results strongly suggested that miR-144 may exert a tumor-suppressive function by repressing the expression of SMAD4 in HCC development.

\section{Conclusion}

The results of this study strongly suggested the tumorsuppressive role of miR-144 in HCC. Moreover, the present study also demonstrated that upregulation of miR-144 leads to inhibition of cell proliferation, cell cycle progression, chemoresistance, and other malignant biological behaviors.

\section{Acknowledgments}

This study was supported by grants from the National Science Foundation of Guangdong Province, People's Republic of China (No 2014A030310073), Guangdong Provincial Science and Technology Plan projects (No 2009B080701021 and 2010B080701021), Guangdong Province Public Interest Research and Capacity - Building Projects, People's Republic of China (No 2014A020212448), and Guangzhou Science and Technology Plan of Scientific Research Projects, People's Republic of China (No 201510010286).

\section{Disclosure}

The authors report no conflicts of interest in this work. 


\section{References}

1. Shen G, Lin Y, Yang X, Zhang J, Xu Z, Jia H. MicroRNA-26b inhibits epithelial-mesenchymal transition in hepatocellular carcinoma by targeting USP9X. BMC Cancer. 2014;14:393.

2. Hou B, Jian Z, Chen S, Ou Y, Li S, Ou J. [Expression of miR-216a in pancreatic cancer and its clinical significance]. Nan Fang Yi Ke Da Xиe Xue Bao. 2012;32(11):1628-1631. Chinese.

3. Yang J, Han S, Huang W, et al. A meta-analysis of microRNA expression in liver cancer. PLoS One. 2014;9(12):e114533.

4. Vosa U, Kolde R, Vilo J, Metspalu A, Annilo T. Comprehensive metaanalysis of microRNA expression using a robust rank aggregation approach. Methods Mol Biol. 2014;1182:361-373.

5. Wu J, Lu P, Yang T, Wang L. Meta-analysis of the differentially expressed breast cancer-related microRNA expression profiles. JObstet Gynaecol. 2014;34(7):630-633.

6. Hong TH, Park IY. MicroRNA expression profiling of diagnostic needle aspirates from surgical pancreatic cancer specimens. Ann Surg Treat Res. 2014;87(6):290-297.

7. Sun Y, Guo F, Bagnoli M, et al. Key nodes of a microRNA network associated with the integrated mesenchymal subtype of high-grade serous ovarian cancer. Chin J Cancer. 2015;34(1):28-40.

8. Tsai MM, Wang CS, Tsai CY, et al. Potential diagnostic, prognostic and therapeutic targets of microRNAs in human gastric cancer. Int $J$ Mol Sci. 2016;17(6):945.

9. Lujambio A, Lowe SW. The microcosmos of cancer. Nature. 2012; 482(7385):347-355.

10. Hashemi GA, Burkhard FC, Rehrauer H, Aquino Fournier C, Monastyrskaya K. MicroRNA MiR-199a-5p regulates smooth muscle cell proliferation and morphology by targeting WNT2 signaling pathway. J Biol Chem. 2015;290(11):7067-7086.

11. Yang R, Chen Y, Tang C, et al. MicroRNA-144 suppresses cholangiocarcinoma cell proliferation and invasion through targeting platelet activating factor acetylhydrolase isoform 1b. BMC Cancer. 2014;14:917.

12. Zhao M, Huang J, Gui K, et al. The downregulation of miR-144 is associated with the growth and invasion of osteosarcoma cells through the regulation of TAGLN expression. Int J Mol Med. 2014;34(6): $1565-1572$.

13. Guo Y, Ying L, Tian Y, et al. miR-144 downregulation increases bladder cancer cell proliferation by targeting EZH2 and regulating Wnt signaling. FEBS J. 2013;280(18):4531-4538.

14. Guan H, Liang W, Xie Z, et al. Down-regulation of miR-144 promotes thyroid cancer cell invasion by targeting ZEB1 and ZEB2. Endocrine. 2015;48(2):566-574.
15. Cao T, Li H, Hu Y, Ma D, Cai X. miR-144 suppresses the proliferation and metastasis of hepatocellular carcinoma by targeting E2F3. Tumour Biol. 2014;35(11):10759-10764.

16. Torbenson M, Marinopoulos S, Dang DT, et al. Smad4 overexpression in hepatocellular carcinoma is strongly associated with transforming growth factor beta II receptor immunolabeling. Hum Pathol. 2002; 33(9):871-876.

17. Yakicier MC, Irmak MB, Romano A, Kew M, Ozturk M. Smad2 and Smad4 gene mutations in hepatocellular carcinoma. Oncogene. 1999; 18(34):4879-4883.

18. Lee DK, Park SH, Yi Y, et al. The hepatitis B virus encoded oncoprotein $\mathrm{pX}$ amplifies TGF-beta family signaling through direct interaction with Smad4: potential mechanism of hepatitis B virus-induced liver fibrosis. Genes Dev. 2001;15(4):455-466.

19. Rossmanith W, Schulte-Hermann R. Biology of transforming growth factor beta in hepatocarcinogenesis. Microsc Res Tech. 2001;52(4): 430-436.

20. Xu Z, Ramachandran S, Gunasekaran M, et al. MicroRNA-144 dysregulates the transforming growth factor-beta signaling cascade and contributes to the development of bronchiolitis obliterans syndrome after human lung transplantation. J Heart Lung Transplant. 2015; 34(9):1154-1162.

21. Ang CW, Nedjadi T, Sheikh AA, et al. Smad4 loss is associated with fewer S100A8-positive monocytes in colorectal tumors and attenuated response to S100A8 in colorectal and pancreatic cancer cells. 2010;31(9): $1541-1551$.

22. Hahn SA, Hoque AT, Moskaluk CA, et al. Homozygous deletion map at 18q21.1 in pancreatic cancer. Cancer Res. 1996;56(3):490-494.

23. Argani P, Shaukat A, Kaushal M, et al. Differing rates of loss of DPC4 expression and of $\mathrm{p} 53$ overexpression among carcinomas of the proximal and distal bile ducts. Cancer. 2001;91(7):1332-1341.

24. Miyaki M, Kuroki T. Role of Smad4 (DPC4) inactivation in human cancer. Biochem Biophys Res Commun. 2003;306(4):799-804.

25. Yamazaki K, Masugi Y, Sakamoto M. Molecular pathogenesis of hepatocellular carcinoma: altering transforming growth factor-beta signaling in hepatocarcinogenesis. Dig Dis. 2011;29(3):284-288.

26. Lu Y, Wu LQ, Li CS, Wang SG, Han B. Expression of transforming growth factors in hepatocellular carcinoma and its relations with clinicopathological parameters and prognosis. Hepatobiliary Pancreat Dis Int. 2008;7(2):174-178.

27. Hiwatashi K, Ueno S, Sakoda M, et al. Strong Smad4 expression correlates with poor prognosis after surgery in patients with hepatocellular carcinoma. Ann Surg Oncol. 2009;16(11):3176-3182.
OncoTargets and Therapy

\section{Publish your work in this journal}

OncoTargets and Therapy is an international, peer-reviewed, open access journal focusing on the pathological basis of all cancers, potential targets for therapy and treatment protocols employed to improve the management of cancer patients. The journal also focuses on the impact of management programs and new therapeutic agents and protocols on
Dovepress

patient perspectives such as quality of life, adherence and satisfaction. The manuscript management system is completely online and includes a very quick and fair peer-review system, which is all easy to use. Visit http://www.dovepress.com/testimonials.php to read real quotes from published authors. 